\title{
The behavior of the sextic coupling for the scalar field at the intermediate and strong coupling regime
}

\author{
Gino J. Añaños \\ Instituto de Física Teórica-IFT \\ Universidade Estadual Paulista \\ Rua Pamplona 145, São Paulo, SP 01405-900 Brazil \\ E-mail:gananos@ift.unesp.br
}

\begin{abstract}
We study the behavior of the renormalized sextic coupling at the intermediate and strong coupling regime for the $\varphi^{4}$ theory defined in $d=2$-dimension. We found a good agreement with the results obtained by the field-theoretical renormalization-group in the Ising limit. In this work we use the lattice regularization method.
\end{abstract}




\section{Introduction}

The scalar field $\left(\varphi^{4}\right)_{d=2}$ theory is known to have an interactive continuum limit. This theory is an excellent theoretical laboratory in which nonperturbative methods can be tested. For theories in less than four space-time dimensions can offer interesting and complex behavior as well as tractability, and for example the case of three space-time dimensions, they can even be directly physical, describing various planar condensed matter systems. The $\varphi^{4}$ theory has been study in the regimen of the intermediate and strong coupling analytically using the path integral representation of the generating functional of the Green's function. Two of these methods are the high temperature series approach of Baker and Kincaid [1] and the strong coupling expansion of Bender et al $[2,3]$. Each of these methods is based in the replacing the continuum by a Euclidean lattice and expanding the kinetic energy. For example in the strong coupling expansion the method consists in expanding the Green's functions in the inverse powers of the bare coupling. Of course the result of this expansion will be better as the bare coupling is strong enough. This is because the expansion requires that the coupling constant has to be large to perform the analytic expansion of the path integral. Another nonperturbative analytic method which was proved to be a powerful tool is the the field theoretical renormalization group [4].

The first study of the $\varphi^{4}$ theory in the regimen of strong coupling using lattice Monte Carlo simulation was done by Cooper, Freedman and Preston [5]. They showed that the $\varphi^{4}$ theory has a non zero scattering amplitude in one and two dimensions and the asymptotic value for the renormalized coupling constant was in good agreement with the high temperature series and strong 
coupling expansion method. Freedman et al [6] continued the study in three and four dimensions and they found Monte Carlo evidence that the lattice $\left(\varphi^{4}\right)_{d=4}$ theory has a noninteracting continuum limit consistent with the result of renormalization group calculation [4] and strong coupling expansion $[2,3]$. In contrast the $\left(\varphi^{4}\right)_{d=3}$ theory appears to have an interacting continuum limit. In this paper we are interested in the higher order renormalized coupling constant in particular the sextic coupling constant $g_{6}$. We expect that in the limit of strong coupling this quantity will approach the asymptotic limit. This because the theory in the strong coupling limit can be considered to be in the the critical region where fluctuations are so strong that they complete screen out the initial interaction so that the behavior of the system becomes universal. In two dimensions, Sokolov and Orlov used renormalization group expansion and Padé-Borel-Leroy resumation technique to get $g_{6}[8]$. In the literature there are few works available of the sextic coupling constant using the approach of lattice Monte Carlo. The first work was performed by Wheter [9] and later by Tsypin [10]. So it seems appropriate the nonperturbative study using the lattice Monte Carlo technique [11] to get not only the asymptotic value of the higher order renormalized coupling constant but also the behavior on the intermediate and strong regimen. Here as usual we consider $\hbar=c=1$. 


\section{The effective potential}

We consider here the $\phi^{4}$ theory in d-dimensions Euclidean space in the presence of a source $J$, which the bare action is given by:

$$
S[\phi, J]=\int d^{d} x\left[\frac{1}{2}\left(\partial_{\mu} \phi_{0}\right)^{2}+\frac{1}{2} m^{2} \phi_{0}^{2}+\frac{g}{4 !} \phi_{0}^{4}-J_{0} \phi_{0}\right]
$$

We introduce the vacuum persistent function $Z[J]$ :

$$
Z[J]=\int D[\phi] e^{-S[\phi, J]}
$$

and from this we obtain the connected n-point Green's functions $G_{n}\left(x_{1} \ldots x_{n}\right)$

$$
G_{n}\left(x_{1} \ldots x_{n}\right)=\left.\frac{\delta}{\delta J\left(x_{1}\right)} \ldots \frac{\delta}{\delta J\left(x_{n}\right)} \ln Z(J)\right|_{J=0}
$$

The renormalized $\Gamma^{(N)}(0)$ proper $N$-point Green's function is obtained as follows: the wavefunction renormalization is obtained from the two-point Green's function from:

$$
Z^{-1}=\left.\frac{G_{2}\left(p^{2}\right)}{d p^{2}}\right|_{p^{2}=0}
$$

and the renormalized mass $m_{r}^{2}$ is defined by

$$
m_{r}^{2}=\left.Z G_{2}^{-1}\left(p^{2}\right)\right|_{p^{2}=0}
$$

The effective action $\Gamma\left[\phi_{c}\right]$ is defined by a functional Legendre transform of $W[J]=\ln Z[J]$ :

$$
\Gamma\left[\phi_{c}\right]=\int d^{d} x \phi_{c}(x) J(x)-W[J]
$$


It is well known that the effective action is the generator of proper Green's functions and in particular we can write the effective action in the form

$$
\Gamma\left[\phi_{c}\right]=\sum_{n} \frac{1}{n} \int d^{d} x_{1} \ldots d^{d} x_{n} \Gamma^{(n)}\left(x_{1}, \ldots, x_{n}\right) \phi_{c}\left(x_{1}\right) \ldots \phi_{c}\left(x_{n}\right)
$$

Here $\Gamma^{(n)}\left(x_{1}, \ldots, x_{n}\right)$ is the proper $n$-point Green's functions in presence of the source $J$. We define the effective potential, $U(\phi)$, by

$$
\Gamma[\phi]=\int d^{d} x\left[U(\phi)+\frac{1}{2}\left(\partial_{\mu} \phi\right)^{2} Z+\ldots\right] .
$$

an the renormalized effective potential

$$
U_{r}=\sum_{n=1}^{\infty} \frac{\Gamma_{r}^{(2 n)}(0)}{2 n !} \phi_{r}^{2 n}
$$

where $\phi_{r}=\phi Z^{-1 / 2}$ and the renormalized $\Gamma^{(n)}(0)$ proper $n$-point Green's functions are given by

$$
\Gamma_{r}^{(n)}(0)=Z^{n / 2} \Gamma^{(n)}(0)
$$

from here we see that $U_{r}$ is the generating function of one particle irreducible renormalized Green's function at zero external momentum on all legs.

The particular interest to us are the renormalized coupling constant $\widetilde{\Gamma}_{r}^{(4)}(0)$ and the renormalized sextic coupling constant $\widetilde{\Gamma}_{r}^{(6)}(0)$ which can be expressed as follows:

$$
\widetilde{\Gamma}_{r}^{(4)}(0)=-\left.Z^{2}\left(\widetilde{G}_{2}^{-1}\right)^{4} \widetilde{G}_{4}\right|_{p=0}
$$

and

$$
\widetilde{\Gamma}_{r}^{(6)}(0)=-\left.Z^{3}\left(\widetilde{G}_{2}^{-1}\right)^{6}\left(\widetilde{G}_{6}-10 \widetilde{G}_{4}^{2} \widetilde{G}_{2}^{-1}\right)\right|_{p=0} .
$$


The quantity that will be extracted from lattice Monte Carlo simulation is the dimensionless renormalized constant $g_{2 n}$ defined by,

$$
g_{2 n}=\frac{\Gamma_{r}^{(2 n)}(0)}{m_{r}^{2 n-n d+d}(2 n) !} .
$$

For $d=2$ this expression becomes

$$
g_{2 n}=\frac{\Gamma_{r}^{(2 n)}(0)}{m_{r}^{2}(2 n) !}
$$

and the effective potential divided by $m_{r}^{2}$ can be written,

$$
U_{r}\left(\phi_{r}\right) / m_{r}^{2}=\frac{1}{2} \phi_{r}^{2}+g_{4} \phi_{r}^{4}+g_{6} \phi_{r}^{6}+\ldots
$$

\section{Simulations results}

For the Monte Carlo simulation of the lattice field theory described by Eq. (2) we used the standard Metropolis algorithm. In order to avoid the trapping into meta stable states due to the underlying Ising dynamics we use the cluster algorithm using the embedded dynamics for $\phi^{4}$ theory, according to the action, [7].

$$
S_{\text {Ising }}=-\sum_{x} \sum_{\hat{\mu}}|\phi(x+\hat{\mu}) \phi(x)| s(x+\hat{\mu}) s(x),
$$

where $s(x)=\operatorname{sign}(\phi(\mathrm{x}))$. Statistical errors are evaluated taking into account the autocorrelation time in the statistical sample generated by the Monte Carlo simulation. In the simulation we used the lattices $16^{2}, 20^{2}, 36^{2}$ and $64^{2}$. To minimize finite size effects we choose $1<<1 /\left(a m_{r}\right)<<L$. For the purpose of numerical simulation it is appropriated to work with equivalence Lagrange on 
the lattice

$$
S[\phi]=2 \kappa \sum_{x, \mu} \phi(x) \phi\left(x+e_{\mu}\right)+\sum_{x} \phi(x)^{2}+\sum_{x} \lambda\left(\phi(x)^{2}-1\right)^{2} .
$$

The parameters $m^{2}$ and $g^{2}$ are related to $\kappa$ and $\lambda$ as follows,

$$
m^{2}=\frac{1-2 \lambda}{\kappa}-2 d \quad g=\frac{6 \lambda}{\kappa^{2}} .
$$

We found the bar parameters $\left(m^{2}, g\right)$ for the different lattices by fixing the value of $\lambda$ and varying the parameter $\kappa$ until we find an acceptable value of the renormalized mass within few percent. All the values of bare mass were negative. Of course we can not have a phase transition since we imposed the renormalized mass to the condition $1<<1 /\left(a m_{r}\right)<<L$. The renormalized mass $m_{r}$ was calculated using the expression

$$
a^{-2} m_{r}^{-2}=\left(\frac{L}{2 \pi}\right)^{2}\left[\frac{<\tilde{\phi}(0)^{2}>-<|\tilde{\phi}(p)|^{2}>}{<|\tilde{\phi}(p)|^{2}>}\right]
$$

where here $\tilde{\phi}$ is the Fourier transform of $\phi$ and $p=\left(\frac{2 \pi}{L a}, 0\right)$ is the smallest available non-zero momentum.

For purpose of calculating the values of $g_{4}$ and $g_{6}$ from simulation we use lattice version of eqs.(10) and (11),

$$
\begin{gathered}
g_{4}(4 !)=\frac{\widetilde{\Gamma}_{r}^{(4)}(0)}{m_{r}^{2}}=-m_{r}^{2} \frac{<\widetilde{\phi}(0)^{4}>-3<\widetilde{\phi}(0)^{2}>^{2}}{<\widetilde{\phi}(0)^{2}>^{2}}, \\
g_{6}(6 !)=\frac{\widetilde{\Gamma}_{r}^{(6)}(0)}{m_{r}^{2}} \\
=10\left(\frac{\Gamma_{r}^{(4)}(0)}{m_{r}^{2}}\right)^{2}-\left(m_{r}^{2}\right)^{2} \frac{<\widetilde{\phi}(0)^{6}>-15<\widetilde{\phi}(0)^{4}><\widetilde{\phi}(0)^{2}>+30<\widetilde{\phi}(0)^{2}>^{3}}{<\widetilde{\phi}(0)^{2}>^{3}},
\end{gathered}
$$




\begin{tabular}{|c|c|c|}
\hline $\mathrm{L}$ & $g_{4}$ & $g_{6}$ \\
\hline 16 & $0.60 \pm 0.013$ & $1.18 \pm 0.06$ \\
20 & $0.60 \pm 0.011$ & $1.16 \pm 0.04$ \\
36 & $0.59 \pm 0.012$ & $1.12 \pm 0.05$ \\
64 & $0.585 \pm 0.012$ & $1.112 \pm 0.04$ \\
\hline
\end{tabular}

Table 1:

where $<\widetilde{\phi}(0)>$ is the Fourier transform evaluated at zero momentum . We notice that eqs.(19) and (20) are efficient in the intermediate and strong coupling regime where the statistical errors are reasonable contrarily to the case of weak coupling. In table 1 . we present the results of simulations for various lattices size for a limit of strong coupling. The results in table 1. are consistent with the values obtained by the field-theoretical renormalization-group in the Ising limit $g_{4}=0.6125$ and $g_{6}=1.10$ [8]. In Fig. 1. we present the results of simulation for $g_{6}$ and $g_{4}$ for a large range of the bare coupling constant $(g \sim 15-3232)$. We observe both $g_{4}$ and $g_{6}$ approach to asymptotic constant value as we expected. From the figure also we can see that the variation of both quantities are small considering the large variation on $g$.

\section{Conclusion}

In this paper we analyzed the behavior of the renormalized sextic coupling for the theory $\left(\phi^{4}\right)_{d=2}$ on the lattice at strong coupling constant. We found that this quantity tend to constant value 


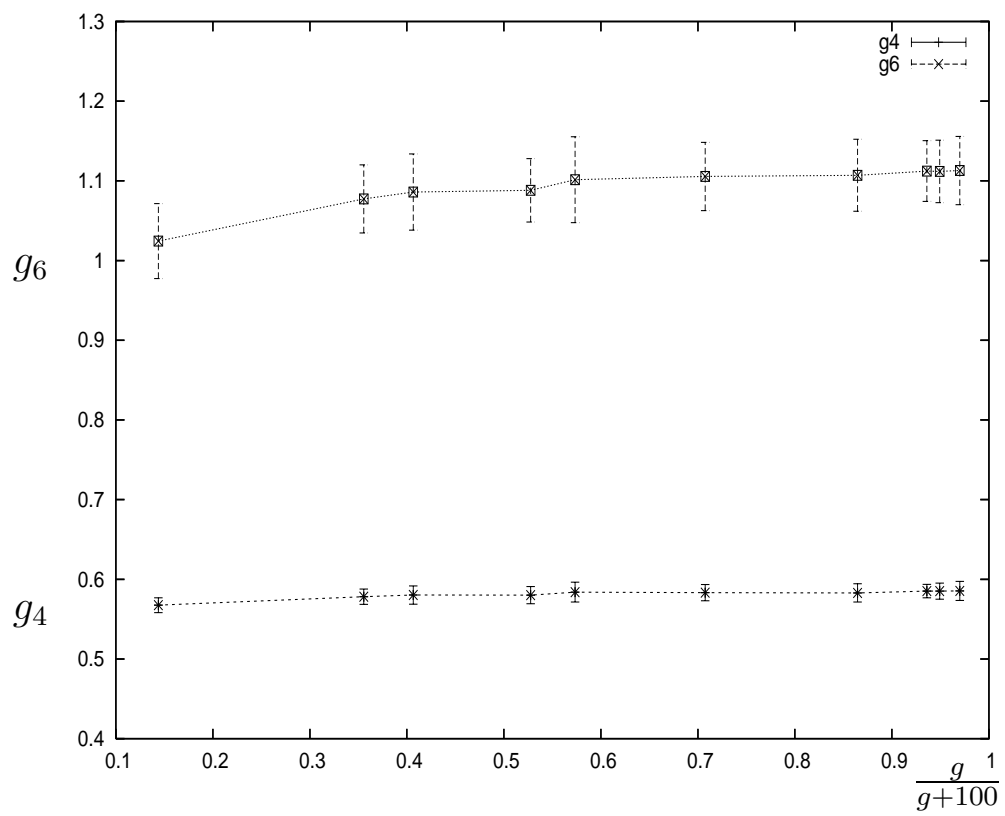

Figure 1: The behavior of $g_{4}$ and $g_{6}$ with the bare coupling constant

that is in good agreement with value obtained by using the field-theoretical renormalization-group in the Ising limit at four loops. We also notice that the qualitative behavior of $g_{6}$ is similar to $g_{4}$. We expect that this behavior is also similar for the case $d=3$, which will be presented on future report. 


\section{Acknowlegements}

This paper was supported by FAPESP under contract number 03/12271-7

\section{References}

[1] G. A. Baker and J. Kincaid, J. Stat. Phys 24, 469 (1981); Phys. Rev. Lett. 42, 1431 (1979).

[2] C. M. Bender, F. Cooper, G.S. Guralnik and D. Sharp Phys. Rev. D 19, 1865 (1979).

[3] C.M. Bender, F. Cooper, G.S. Guralnik and D. Sharp, Phys. Rev. D 23, 2976 (1981).

[4] K.Wilson, Phys.Rev. B4, 3184 (1971).

[5] F. Cooper, B. Freedman and D. Preston, Nucl. Phys. B 210, 210 (1982).

[6] B. Freedman, P. Smolensky and D. Weingarten, Phys. Lett. B 113, 481 (1982).

[7] R. C. Brower and P. Tamayo, Phys. Rev. Lett. 62, 1087 (1989).

[8] A. I. Sokolov and E. V. Orlov, Phys. Rev. B 58, 2395 (1998) [arXiv:cond-mat/9804008].

[9] J.F Wheter, Phys. Lett. 136B, 402 (1983).

[10] M.M Tsypin, Phys. Rev. Lett. 73, 2015 (1994).

[11] I. Montvay, I. Munster, Quantum fields on the lattice, (University of Cambridge Press, 1994). 